\title{
Software-hardware Complex of Access Control and Management
}

\author{
Lytvynenko Vladyslav $^{1}$ \\ Ivashchenko Heorhii $^{2}$
}

${ }^{1}$ Kharkiv National University of Radio Electronics, 14 Nauky Ave, KharkivUA-61166, Ukraine, vladyslav.lytvynenko@nure.ua ${ }^{2}$ Kharkiv National University of Radio Electronics, 14 Nauky Ave, KharkivUA-61166, Ukraine, heorhii.ivashchenko@nure.ua

\begin{abstract}
The presented work is dedicated to the issues of development software-hardware complex of access control and management based on Arduino platform. The hardware part of the complex works together with the web-application, created using React, Redux, Node.js and MongoDB technologies. This provides extensive functionality of the application.
\end{abstract}

Keywords: access control, RFID, Node MCU, React, Node.js, MongoDB.

\section{INTRODUCTION AND PROBLEM STATEMENT}

In commercial and industrial settings, the issue of access control of identified individuals to various buildings and premises is one of a high-priority. The possibility of control comprises the prevention of access by the mechanism responsible for the functions of locking and unlocking.

Management of access control system allows to change the privileges of selected individuals and record all attempts of entrance. The use of RFID tags, which may be implemented in different formats, became a common method of user identification.

Comparison of existing systems of access control and management (SACM) revealed a range of their drawbacks: high cost, limited functionality, inability to save data on the personal server, limited ability to activate/deactivate access keys without rewriting software, complicated management of users' RFID tags status.

\section{PROPOSED SOLUTION}

The solution of the issue of access control and management is development of competitive softwarehardware complex, which provides versatility, as well as the ability to broaden functionality.

Proposed solution is based on the use of hardware platform Arduino. Module RC522 is used to read data from an RFID tag. NodeMCU platform is used to process and send RFID tag data to the server. This platform is also a controlling mechanism (with the use of switching relay and solenoid) for granting access. This platform represents a board for development based on ESP8266 (version ESP12E) and includes UART-Wi-Fi module with low energy consumption [1].

Performance of switching relay and solenoid depends on power availability, in the case of absence of which, the complex is not able to function properly and remains in a closed state. This allows to prevent trespassing onto a premises by shutting down the power grid.

Web-application is used for managing the hardware part of the complex, server side of which is responsible for the business logic of SACM solution, and is implemented on a programming platform Node.js using Express framework [2].
Interaction with the hardware part takes place with the help of HTTP request that is sent from Node MCU platform to the server, which processes received data and sends back a response. The use of Node.js platform together with Socket.io library provides convenient application of WebSocket communications protocol, which allows transferring data from the server without waiting for a request from the client side.

Data is saved by means of non-relational database management system MongoDB, to work with which ODM (Object Data Modelling) library Mongoose is used. This ensures safety, simple integration and high productivity of data manipulation [3].

Client side is implemented in the form of an SPAapplication, by means of which administrator can control access and view logs by graphic interface [3]. Administrator can add new users to the system and change status of existing RFID tags, which have been used at least once for an attempt of entrance. The developed solution allows to collect data about all entrance attempts regardless of their result or whether the users are in the database and export of data in CSV format for further processing.

Client side was created with the React library, used for making user interfaces and intended to solve issues of partial renewal of web-page content [4]. For controlling the state of the developed SPA-application, React is used in combination with Redux library. Redux enables saving the state of the whole application in the tree of objects in a single storage.

\section{CONCLUSIONS}

The developed solution for access control and management encompasses software part and hardware part. Software part is in the form of a web-application which combines simple graphic user interface, support of necessary functionality and the possibility of its expansion. Hardware part is implemented on NodeMCU platform containing a microcontroller, Wi-Fi module, which is well suited for creating Internet of Things.

Using the suggested complex will provide the flexibility of setting access modes, logging and controlling working hours, higher level of safety and a possibility to receive information regarding whether employees adhere to the work schedule or not.

\section{REFERENCES}

[1] M. Jayakumar, "The internet of things with esp8266 Hands on approach: Get started with Arduino IDE and ESP8266," CreateSpace Independent Publishing Platform, 2017, 270 p.

[2] G. Lim, "Beginning Node.js, Express \& MongoDB Development," Independently published, 2019, $151 \mathrm{p}$.

[3] H. San, "MongoDB Best Practices: Build Fault Tolerant Applications," Kindle Edition, 2014, 131 p.

[4] A. Banks, E. Porcello, "Learning React: Functional Web Development with React and Redux," Apress, 2017, 350 p. 